\title{
ESTADO DEL ARTE MPLS-TP CONMUTACIÓN MULTIPROTOCOLO MEDIANTE ETIQUETAS - PERFIL DE TRANSPORTE
}

\section{STATE OF THE ART MPLS-TP (SWITCHING MULTI- PROTOCOL USING TAGS - TRANSPORT PROFILE)}

\begin{abstract}
The increasing demand in telecommunication services generates the need to develop different network models. In 2008, the ITU$\mathrm{T}$ joins forces with the IETF to develop a protocol called MPLS-TP whose architecture represents the result of the adding of the efficiency in the transport of IP/MPLS packages counting on the reliability of SONET/SDH transport. This paper presents a State of the Art of MPLS-TP where the current studies and advances are reflected in regard to this technology: architecture optical models, OAM protection models.
\end{abstract}

Also presents the structure, functioning and characteristics that make MPLS-TP a good choice for next generation transport networks. The material support for the development of this article has been obtained from the documentary basis of electronic resources with scientific content supporting the investigation of the Universidad Distrital Francisco José de Caldas.

Key words: administration and management (OAM), multiprotocol label switching transport profile (MPLS-TP), operation, service level agreement (SLA), synchronous digital hierarchy (SDH).

\section{RESUMEN}

La creciente demanda en servicios de telecomunicaciones genera la necesidad de desarrollar diferentes modelos de red. En el año 2008, la UIT-T une sinergias con la IETF para desarrollar un protocolo llamado MPLS-TP cuya arquitectura representa el resultado la agregación de la eficiencia en el transporte de paquetes de IP/ MPLS con la fiabilidad en el transporte de SONET/SDH. Este trabajo presenta un estado del arte de MPLS-TP donde se plasman los estudios actuales y avances relacionados con ésta tecnología: modelos de arquitectura óptica, modelos de protección en OAM.

Además se presenta la estructura, funcionamiento y características que hacen de MPLS-TP una opción para las redes de transporte de siguiente generación. El material de apoyo para el desarrollo de este artículo ha sido obtenido de la base documental de recursos electrónicos con contenido científico de apoyo a la investigación de la Universidad Distrital Francisco José de Caldas.
Lois Albert Espinel García

Ingeniero de Telecomunicaciones Analista Inalámbrico UNE EPM S.A. albertlois@gmail.com

Bogotá, Colombia

\section{Luis Fernando Pedraza}

Magister en Ciencias de la Información y las Comunicaciones

Docente planta de la Universidad Distrital Francisco José de Caldas lfpedrazam@udistrital.edu.co Bogotá, Colombia

\section{Octavio Salcedo Parra}

Magister en Ciencias de la Información y las Comunicaciones

Docente planta de la Universidad Distrital Francisco José de Caldas ojsalcedop@udistrital.edu.co Bogotá, Colombia

Tipo: Artículo de reflexión

Fecha de Recepción: Julio 8 de 2013 Fecha de Aceptación: Agosto 6 de 2013 
Palabras claves: acuerdo de nivel de servicio (SLA), administración y gestión (OAM), conmutación multiprotocolo mediante etiquetas perfil de transporte (MPLS-TP), jerarquía digital síncrona (SDH), operación.

\section{INTRODUCCIÓN}

Dado que el tráfico de datos comenzó a dominar las redes de telecomunicaciones, se generó la necesidad de una red de transporte para este tipo de tráfico en contraposición a las redes de transporte para TDM. Las tecnologías de transporte tradicionales como SONET, SDH y OTN (recientemente desarrollada) se orientaron para el tráfico de voz. La necesidad de disponer de una tecnología que conserve las mejores características de las tecnologías de transporte diseñadas para la conmutación de paquetes, dio origen a MPLS-TP, como resultado del esfuerzo conjunto de la IETF y la UIT-T [1], la figura 1, ilustra el concepto general de la arquitectura de MPLS-TP con la agregación de la eficiencia en el transporte de paquetes de IP/MPLS con la fiabilidad en el transporte de SONET/SDH.

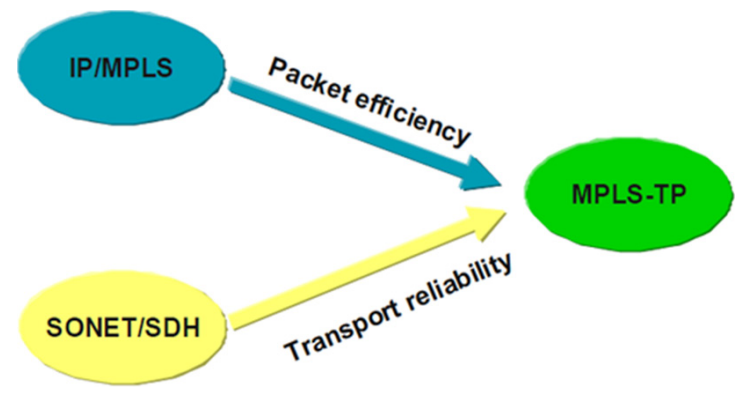

Figura 1. Arquitectura general de MPLS-TP [2]

MPLS-TP utiliza un subconjunto de las normas MPLS donde los elementos que no son requeridos en una red de transporte, como el reenvío de IP, retiro de etiquetas en los paquetes antes de llegar al router frontera (penultimate hop popping PHP), enrutamiento por multiples rutas de igual costo (ECMP) son descartados y no soportados. Por otro lado, MPLS-TP define las extensiones de los estándares MPLS ya existentes e introduce requisitos establecidos en las redes de transporte, incluyendo características de protección y un amplio conjunto de funciones de Operación y Mantenimiento (OAM). Las funciones OAM ayudan en la rápida detección y localización de averías, resolución de problemas, verificación de SLA's y supervisión del rendimiento.

La protección funcional permite el restablecimiento de la red de transporte en tiempos menores a los $50 \mathrm{~ms}$ para topologías de fibra óptica en bus y en anillo. Al proporcionar un amplio conjunto de funciones OAM, herramientas de recuperación y gestión de red (NMS), MPLS-TP permite la operación más determinista de la red con lo cual se consigue la rápida detección y corrección de cualquier violación de un SLA proporcionando a los administradores la simplificación de la operación y el control total de la red, lo que conduce a la reducción operativa de gastos (OPEX) [3].

MPLS-TP tiene algunas características opcionales como la agregación del plano de control, su ausencia no interfiere en las funciones de OAM y de protección gracias al diseño de MPLS-TP. Las figuras 2 y 3 , ilustran el conjunto de funcionalidades generales de MPLS-TP [3].

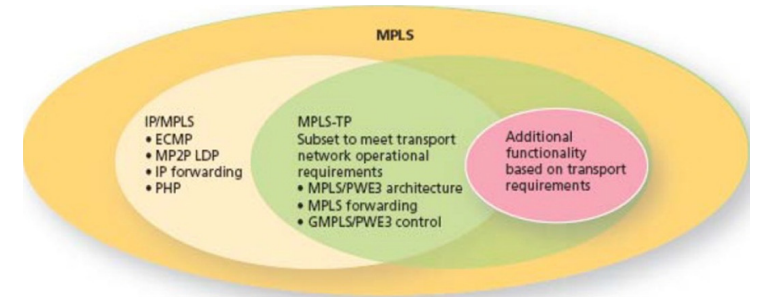

Figura 2. Funcionalidades básicas de MPLS-TP [3]

MPLS-TP presenta características asociadas a las redes de transporte como lo es: protección en la conmutación y un plano de datos, este último basado en OAM y separado del plano de control. 


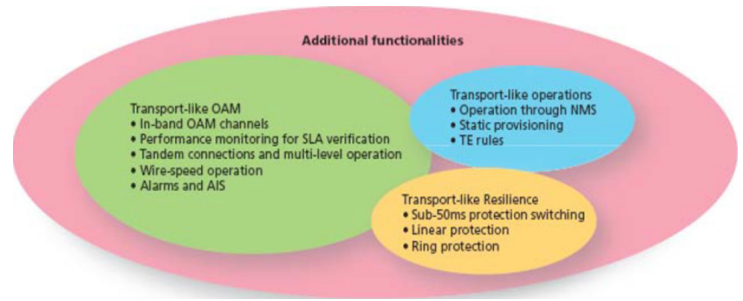

Figura 3. Funcionalidades adicionales de MPLS-TP [3]

Estas características facilitan la transición a una red de transporte de paquetes orientada a la conexión, proporcionando una conmutación de paquetes eficiente, al mismo tiempo que permite las operaciones de transporte de alta calidad.

El presente artículo se enfoca en los nuevos avances y desarrollos relacionados con las nuevas arquitecturas ópticas y nuevos modelos de protección para las funciones de OAM relacionadas con MPLS-TP. En las secciones 1 a 4 se discuten los conceptos básicos de MPLS-TP: evolución, arquitectura y herramientas para OAM. La sección 5 describe el estado del arte para las arquitecturas ópticas y modelos de protección que han sido objeto de evaluación a fin de ser implementados con MPLS-TP, para luego terminar con las conclusiones de este documento.

\section{EVOLUCIÓN HACIA MPLS-TP}

Durante los últimos años se han realizado un conjunto de mejoras a los estándares que definen las redes de transporte, las cuales desempeñan un papel crucial para los portadores de telecomunicaciones y más aún si se tiene en cuenta que las plataformas de servicios dependen de enlaces fiables y estables entre los elementos y nodos de la red. Las redes de transporte como SDH proporcionan enlaces de alta capacidad, permiten la gestión de los medios de enlace físicos, además de garantizar la conectividad entre los recursos de la red con diferentes plataformas [4], sin embargo, las redes de transporte de paquetes proporcionan una mayor flexibilidad y eficiencia que SDH para soportar la próxima generación redes.

La evolución de las redes de transporte hacia
MPLS-TP se ilustra en la figura 4, se observan tres épocas bien diferenciadas (que abarcan desde el 1990 hasta el 2005) y que han sido marcadas por las tecnologías desarrolladas en las redes de transporte, adicionalmente, se observa cómo se han reformulado estas tecnologías para satisfacer los requisitos de los nuevos mercados.

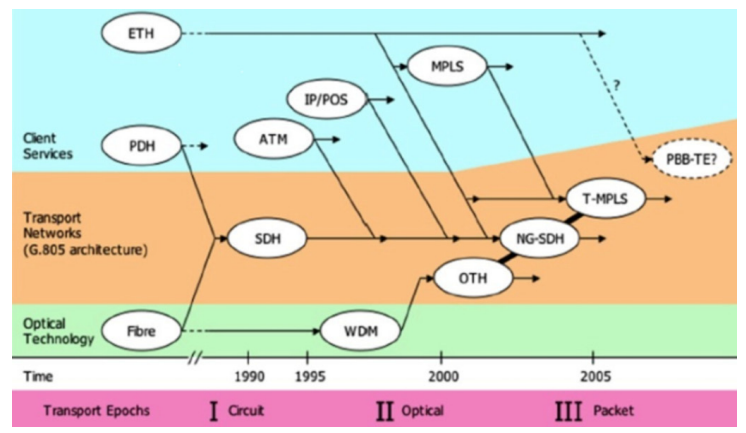

Figura 4. Evolución de las redes de transporte [5]

- Época I transporte por circuito - jerarquía digital síncrona.

- Época II transporte por redes ópticas - jerarquía digital síncrona, jerarquía de transporte óptico.

- Época III transporte por paquetes - redes de siguiente generación, transporte de conmutación multiprotocolo mediante etiquetas, jerarquía digital síncrona de nueva generación [5].

En el año de 1990 SDH fue adoptada por las compañías de telecomunicaciones (carrier's) como la mejor forma de desplegar las redes de transporte de circuitos. Después de diez años (en el año 2000), otra tecnología denominada Multiplexación por División de Longitud de Onda (WDM) resulto de mayor interés debido a la necesidad de satisfacer el incremento en la demanda de servicios.

La Red Óptica de Transporte (OTN), también conocida como el estándar de Jerarquía de Transporte Óptica (OTH), describe un medio de comunicación de datos a través de una red óptica. Dicho estándar fue creado con la intención de combinar los beneficios de la tecnología SONET/SDH con las capacidades de expansión de ancho de banda que ofrece la tecnología de 
Multiplexación por División en Longitudes de Onda Densas (DWDM) [6].

El IETF desarrolló originalmente el protocolo MPLS a fin de abordar los problemas de rendimiento de los routers en el núcleo (backbone) IP. Mientras que las redes de conmutación de paquetes se fue incrementando, la UIT-T se interesó en la adaptación de MPLS como red de transporte a nivel de portador (carrier) con las funciones y principios de arquitectura definidos en las recomendaciones UIT-T para las redes de transporte.

Por esta razón, el resultado fue la arquitectura MPLS de transporte (T-MPLS), como red de transporte de paquetes orientada a la conexión basada en MPLS cuyo principio es proporcionar conexiones gestionadas punto a punto para diferentes redes en la capa del cliente.

MPLS-TP tiene sus inicios en MPLS-T de la UIT$\mathrm{T}$, y que luego paso a denominarse MPLS-TP como resultado del acuerdo alcanzado entre la UIT-T y el IETF [1] con el objeto de producir un conjunto convergente de normas para MPLS-TP con la propiedad de reutilizar la mayor parte de las características de MPLS, MPLS-T, GMPLS, para luego añadir mejoras, sobre todo en el ámbito de la Operación, Administración y Mantenimiento (OAM).

\section{RELACIÓN ENTRE LAS RECOMENDACIO- NES DE LA UIT-T Y LAS RFC DEL IETF}

Sobre la base del trabajo en conjunto (Joint Working Team) JWT de la UIT-T y el IETF, se acordó que el esfuerzo hacia el futuro de la normalización debería centrarse en la definición de MPLS-TP dentro de la IETF con los mismos requisitos funcionales que impulsaron el desarrollo de MPLS-T.

De esta manera, cuando las Peticiones De Comentarios (RFC) de la IETF alcanzaran un nivel de madurez técnicamente comparable a las Recomendaciones existentes sobre MPLS-T; la UIT-T se alinearía a este último con los logros MPLS-TP alcanzados por la IETF [7].
La historia y el proceso para producir un estándar coherente y convergente de MPLS-TP constituido por las RFC's de la IETF y las recomendaciones de la UIT-T se ilustran en la figura 5.

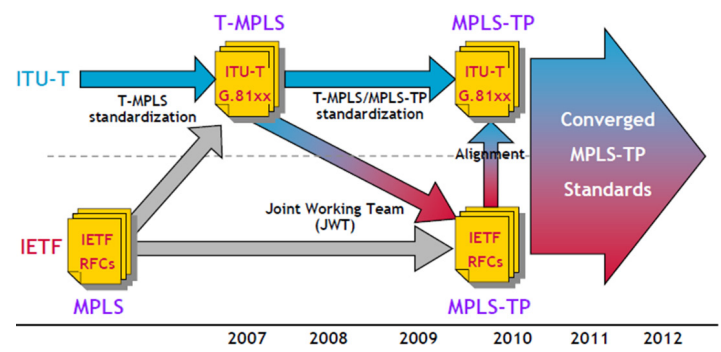

Figura 5. Convergencia hacia un estándar sólido de MPLS-TP [7]

Las especificaciones para MPLS-TP vienen progresando a buen ritmo; la serie de Recomendaciones G.81xx de la UIT-T ya han sentado las bases de MPLS-TP de igual forma ya se cuenta con lanzamientos estables de las especificaciones RFC's de la IETF para MPLS-TP; la figura 6, ilustra la relación entre las recomendaciones de la UIT-T y las peticiones de comentarios del IETF.

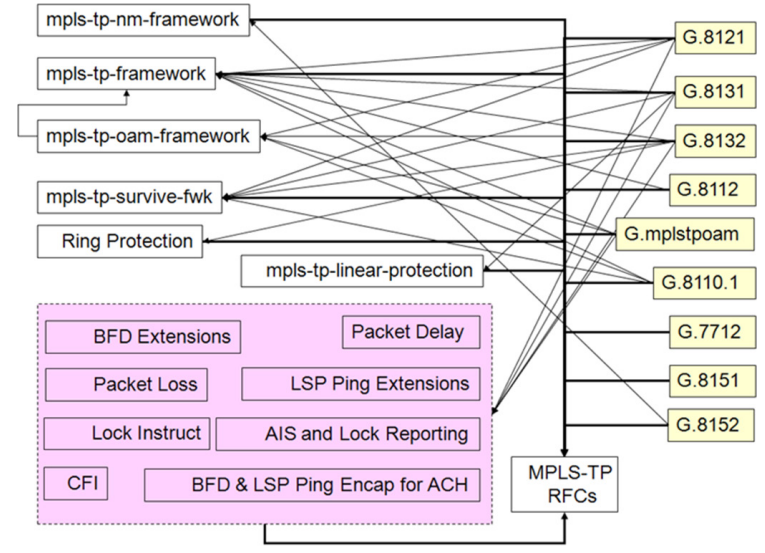

Figura 6. Relación recomendaciones UIT-T y RFC's del IETF [8]

La relación entre las Recomendaciones de la UIT-T y las recomendaciones que se referencian normativamente se ilustran en la figura 7.

G.8101 no se muestra. El desarrollo de G.8101 depende de la finalización de las recomendaciones que utilizarán los términos que se definan. La línea discontinua indica que la versión 
inicial de G.8151 puede no incluir el modelo de protección lineal y/o en anillo. La UIT-T señaló la posibilidad de consentir las recomendaciones en tres bloques [8].

1. G.8110.1, G.7712, G.8112, junio 2010.

2. G.mpls-TP OAM, G.8121, G.8151, febrero 2011.

3. G.8131, G.8132, G.8152, diciembre 2011.

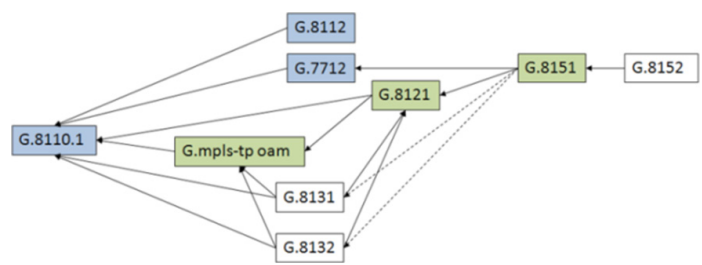

Figura 7. Referencias normativas recomendaciones para MPLS-TP [8]

\section{MPLS-TP}

El objetivo de MPLS-TP es proporcionar servicios de transporte orientado a la conexión para las tecnologías y servicios de conmutación de paquetes (IP) y de conmutación de circuitos (TDM), a través de redes ópticas que aprovechen la tecnología MPLS ampliamente desplegada como se ilustra en la figura 8 . La clave para este esfuerzo es la definición y ejecución de las funciones de OAM y las características de resiliencia para asegurar las capacidades requeridas de las redes de transporte tipo portador [7] como lo es:

- Operaciones escalables.

- Alta disponibilidad.

- Monitorización del rendimiento.

- Soporte multi-dominio.

Las principales características de MPLS-TP [7] son:

- Es estrictamente orientado a la conexión

- Es independiente de requisitos tipo cliente, soporta servicios de capas L3, L2, L1.

- Es independiente de la capa física, se implementa sobre cualquier modelo IEEE, ethernet de PHY (physical layer of the OSI), SONET/SDH [G.783] y OTN [G.709], [G.872] utilizando GFP [G.7041], WDM, DWDM, etc.
- Proporciona fuertes funciones de OAM similares a las disponibles en las redes ópticas de transporte tradicionales como SONET/SDH, OTN, como parte integral del plano de datos de MPLS-TP y son independientes del plano de control.

- Proporciona varios esquemas de protección en el plano de datos similares a los disponibles en las redes ópticas de transporte tradicionales.

- Permite el aprovisionamiento de la red, a través de un NMS centralizado y/o un plano de control distribuido.

- El plano de control GMPLS es también aplicable a las capas de cliente o servidor de MPLS-TP y permite utilizar un enfoque común para la gestión y el control de las múltiples capas de las redes de transporte.

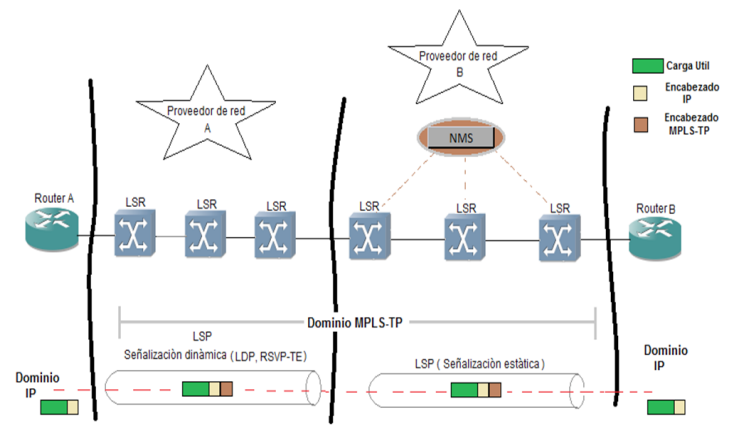

Figura 8. Transporte orientado a la conexión en MPLSTP [9]

\subsection{Arquitectura de MPLS-TP}

MPLS-TP (figura 8) conserva la arquitectura de red y un conjunto de protocolos compatibles con MPLS, introduciendo funciones adicionales de OAM con el objeto de mejorar la detección y gestión fallas.

Estos protocolos y nuevas mejoras se pueden separar en las siguientes categorías:

- Arquitectura de red: cubre la definición de varias funciones y las interacciones entre ellas.

- Plano de datos: cubre los protocolos y mecanismos que se utilizan para transmitir y/o enviar los paquetes de datos.

- Plano de control: cubre los protocolos y 
mecanismos utilizados para establecer los caminos de conmutación de etiquetas (LSP) que se utilizan para reenviar los paquetes de datos.

- Plano de gestión: cubre los protocolos y mecanismos que se utilizan para gestionar la red.

Una lista de protocolos y mecanismos en cada una de estas categorías se ilustran en la figura 9, también se destaca el conjunto de mejoras que están siendo perseguidas por MPLS-TP.

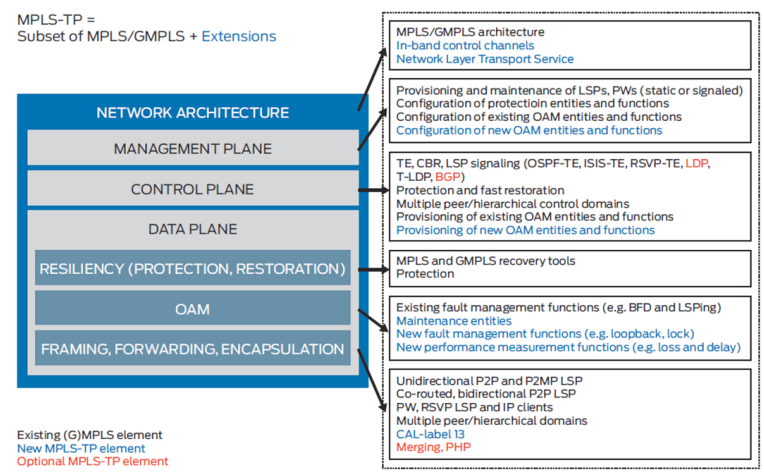

Figura 9. Componentes de MPLS y MPLS-TP [10]

El protocolo y los mecanismos resaltados en azul se añaden del conjunto de protocolos MPLS/GMPLS como parte del esfuerzo de MPLS-TP. Los protocolos y mecanismos resaltados en rojo podrían no ser necesarios para las redes de transporte por lo tanto son opcionales.

La correspondencia de la arquitectura de MPLSTP con el modelo de referencia OSI se ilustra en la figura 10.

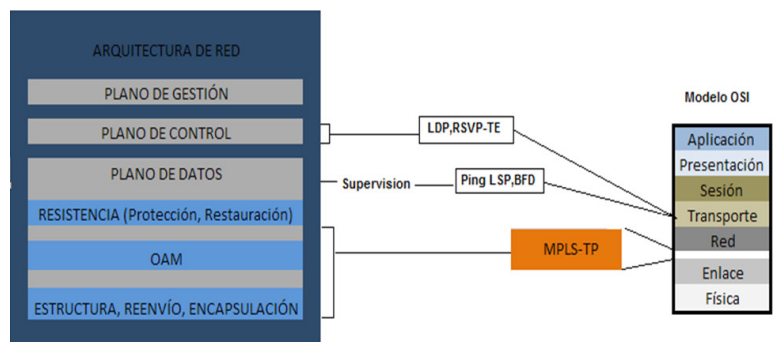

Figura 10. Relación arquitectura de MPLS-TP en el modelo OSI [9]

A diferencia de las tecnologías de modo de transferencia asíncrono (ATM) y de la jerarquía digital síncrona, las cuales tienen un número fijo de instancias de capa de red, MPLS-TP soporta un número arbitrario de estas instancias. El número de instancias de capa de red es limitado en la práctica por los alcances físicos (por ejemplo, la MTU de los enlaces físicos subyacentes). MPLS-TP se puede utilizar en un gran número de formas para implementar redes de transporte de paquetes. La estructura de capas que se ilustra en la figura 11, proporciona el modelo de capas de la arquitectura de MPLS-TP que permite estas diferentes implementaciones.

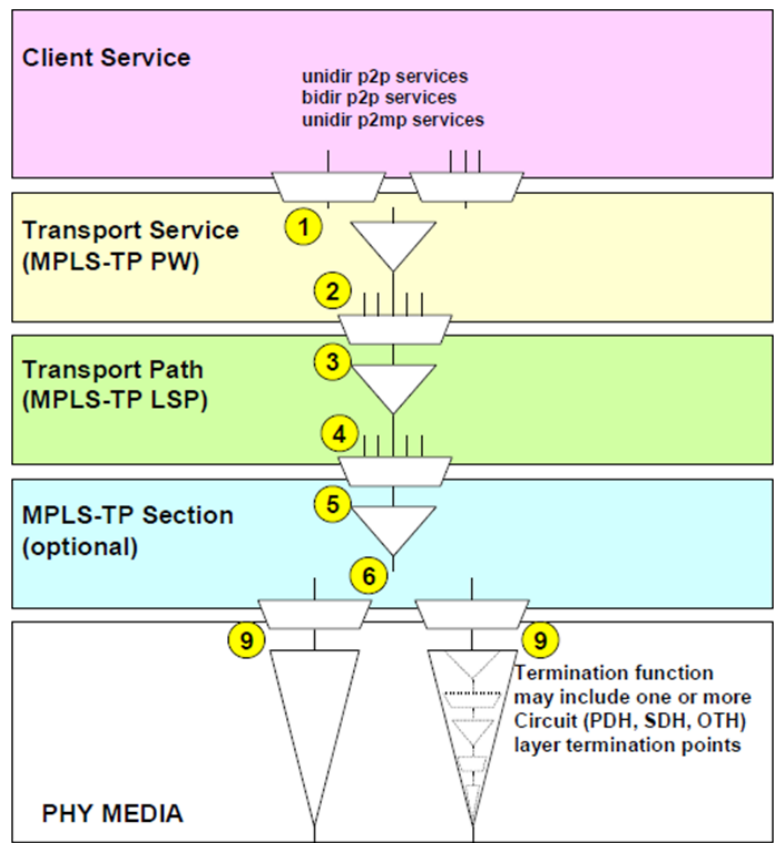

Figura 11. Arquitectura de capas de MPLS-TP [11]

En este modelo de capas se diferencian tres instancias de capa de red: PW, LSP y MTS (como opcional):

- La instancia de la capa de red PW proporciona la capa de servicios de transporte definidos en [IETF RFC 5654] [12], además de la funcionalidad de supervisión inherente de OAM para las conexiones de red que soportan el servicio al cliente.

- La instancia de la capa de red LSP proporciona la capa de trayecto de transporte tal como se define en [IETF RFC 5654] [12]; una conexión de LSP lleva una o más seña- 
les PW entre los bordes de dominios LSP.

- La instancia de la capa de red MTS (opcional) proporciona la capa de sección tal como se define en [IETF RFC 5654] [12]; una conexión MTS porta una o más señales LSP entre los nodos de la red MPLS-TP. La instancia de la capa de red MTS proporciona OAM para el monitoreo de las señales para las conexiones punto a punto de la capa de medios de trasmisión que interconecta los nodos de la red MPLS-TP. MTS como instancia de capa de red opcional, normalmente se usaría en los casos en que la capa de medios físicos no soporte adecuadamente la funcionalidad de OAM requerida [11].

\subsection{Herramientas OAM para MPLS-TP}

El conjunto de herramientas de OAM MPLS-TP está actualmente en proceso de lanzamiento por el IETF y comprenden las características OAM enumeradas en la figura 12 . Los requisitos detallados para las diferentes funciones de OAM se encuentran en los borradores y en las versiones estables de las RFC's de la IETF, un breve resumen se despliega en la tabla 1.

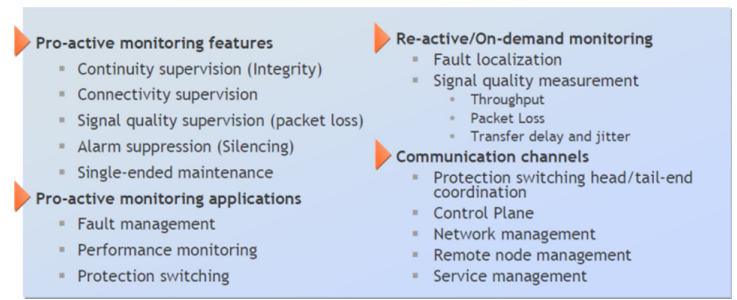

Figura 12. Herramientas OAM para MPLS-TP [7]

El objetivo principal que se pretende, es que los paquetes OAM dedicados se intercalen de manera asociada con en el tráfico de usuario. Estos paquetes de OAM son creados y procesados por un punto de mantenimiento final. Los puntos intermedios de mantenimiento también pueden procesar estos paquetes OAM y pueden recoger datos o alarmas en aumento. Las herramientas se pueden clasificar en las funciones proactivas de OAM que se ejecutan todo el tiempo y en las funciones de monitoreo.

Tabla 1. Características de funciones Oam en mpls-tp [7]

\begin{tabular}{|l|l|}
\hline \multicolumn{1}{|c|}{ RFC / INTERNET-DRAFT } & \multicolumn{1}{c|}{ TITLE } \\
\hline RFC 5317 & $\begin{array}{l}\text { JWT report on MPLS architectural considerations for a transport } \\
\text { profile }\end{array}$ \\
\hline draft-ietf-mpls-tp-requirements-05 & MPLS-TP requirements \\
\hline draft-ietf-mpls-tp-framework-00 & A framework for MPLS in transport networks \\
\hline draft-ietf-mpls-tp-oam-requirements-01 & Requirements for OAM in MPLS transport networks \\
\hline draft-ietf-mpls-tp-oam-framework-00 & MPLS-TP OAM framework and overview \\
\hline draft-ietf-mpls-tp-nm-req-00 & MPLS TP network management requirements \\
\hline draft-ietf-mpls-tp-gach-gal-02 & MPLS generic associated channel \\
\hline draft-ietf-mpls-tp-gach-dcn-00 & An inband data communication network for the MPLS-TP \\
\hline draft-ietf-mpls-tp-gach-dcn-00 & $\begin{array}{l}\text { An inband data communication network for the MPLS transport } \\
\text { profile }\end{array}$ \\
\hline draft-abfb-mpls-tp-control-plane-framework-00 & MPLS-TP control plane framework \\
\hline draft-andersson-mpls-tp-oam-def-01 & "The OAM acronym soup" \\
\hline draft-andersson-mpls-tp-process-00 & $\begin{array}{l}\text { Joint IETF and ITU-T multi-protocol label switching (MPLS) } \\
\text { transport profileprocess }\end{array}$ \\
\hline draft-bhh-mpls-tp-oam-y1731-01 & MPLS-TP OAM based on Y.1731 \\
\hline draft-boutros-mpls-tp-cv-01 & Connection verification for MPLS transport profile LSP \\
\hline draft-boutros-mpls-tp-fault-01 & Fault management for the MPLS \\
\hline draft-boutros-mpls-tp-fault-01 & Fault management for the MPLS transport profile \\
\hline
\end{tabular}




\begin{tabular}{|l|l|}
\hline \multicolumn{1}{|c|}{ RFC / INTERNET-DRAFT } & \multicolumn{1}{c|}{ TITLE } \\
\hline draft-boutros-mpls-tp-loopback-02 & Operating MPLS transport profile LSP in loopback mode \\
\hline draft-boutros-mpls-tp-performance-01 & Performance monitoring of MPLS transport profile lsp \\
\hline draft-bryant-mpls-tp-ach-tlv-01 & Definition of ACH TLV structure \\
\hline draft-ceccarelli-mpls-tp-p2mp-ring-00 & P2MP traffic protection in MPLS-TP ring topology \\
\hline draft-fhbs-mpls-tp-cv-proactive-00 & MPLS-TP proactive continuity and connectivity verification \\
\hline draft-fulignoli-mpls-tp-ais-lock-tool-00 & MPLS-TP OAM alarm suppression tools \\
\hline draft-helvoort-mpls-tp-rosetta-stone-00 & $\begin{array}{l}\text { A thesaurus for the terminology used in multiprotocol label } \\
\text { switchingtransport profile (MPLS-TP) drafts/rfcs and itu-t's } \\
\text { transport network recommendations. }\end{array}$ \\
\hline draft-liu-mpls-tp-bnm-00 & $\begin{array}{l}\text { Multiprotocol label switching transport profile backward notify } \\
\text { messagepacket }\end{array}$ \\
\hline draft-mansfield-mpls-tp-nm-framework-00 & MPLS TP network management framework \\
\hline draft-martinotti-mpls-tp-interworking-01 & Interworking between MPLS-TP and IP/MPLS \\
\hline draft-sprecher-mpls-tp-survive-fwk-01 & $\begin{array}{l}\text { Multiprotocol label switching transport profile survivability } \\
\text { framework }\end{array}$ \\
\hline draft-weingarten-mpls-tp-linear-protection-01 & MPLS-TPlinear protection \\
\hline draft-yang-mpls-tp-ring-protection-analysis-00 & $\begin{array}{l}\text { Multiprotocol label switching transport profile ring protection } \\
\text { analysis }\end{array}$ \\
\hline
\end{tabular}

\subsection{Plano de control para MPLS-TP}

El plano de control de MPLS-TP (figura 13) está definido por la IETF como el plano de MPLS-G para desarrollar un plano de control dinámico que se puede aplicar a la conmutación de paquetes y redes ópticas. La arquitectura MPLS$G$ se describe en [RFC3945]. El plano de control de GMPLS o su equivalente de la UIT-T Red óptica Automáticamente Conmutada (ASON) [G.8080], apoya las funciones de gestión de conexión, así como la protección y restauración, por lo tanto proporciona supervivencia de la red a través de redes compuestas por routers, MPLS LSRs,-TP, ADM's ópticos, conectores cruzados, y dispositivos WDM.

MPLS-TP puede utilizar el plano de control distribuido para permitir la prestación de servicios rápidos, dinámicos y fiables en entornos de múltiples proveedores y múltiples dominios utilizando protocolos estandarizados que garantizan la interoperabilidad.

El plano de control MPLS-TP se basa en una combinación del plano de control MPLS para pseudo-cables y el plano de control GMPLS para MPLS-TP LSP respectivamente como se ilustra en la figura 13. El plano de control MPLSTP distribuido proporciona las siguientes funciones básicas:

\section{- Señalización.}

- Enrutamiento.

- Ingeniería de Tráfico y restricciones basadas en cálculos de ruta.

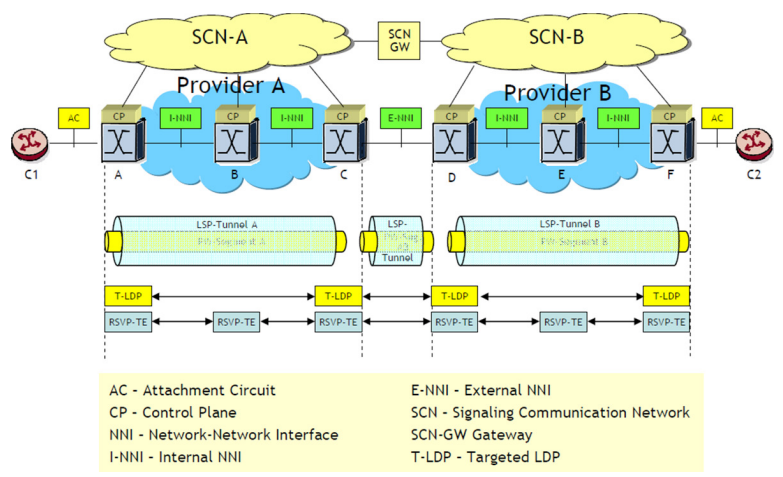

Figura 13. Vista del plano de control de un pseudo-cable multi-segmento [7]

Por otra parte, el plano de control MPLS-TP es capaz de realizar una rápida restauración en el caso de fallas en la red. El plano de control MPLS-TP proporciona características para asegurar su propia supervivencia y lo habilita para 
permitir la recuperación correcta de fallas y deterioros en la red.

El plano de control y el plano de datos de MPLSTP se encuentran lo más desacoplados posibles, de tal manera que las fallas en el plano de control no afecten el plano de datos y viceversa.

\section{ESTADO DEL ARTE MPLS-TP}

En la actualidad existen varios estudios relacionados con nuevas arquitecturas ópticas y nuevos modelos de protección para las funciones de OAM relacionados con MPLS-TP, que buscan generar mayor valor a esta nueva tecnología que se perfila como la red de transporte de paquetes de nueva generación.

\subsection{Arquitectura de redes de transporte ópticas}

Estudios recientes en [13], [14], [15] y [16] están enfocados en las perspectivas y retos que enfrentan las nuevas arquitecturas de red de transporte ópticas de nueva generación donde se incluye MPLS-TP; las cuales deben cumplir con estrictos requisitos en materia de calidad de servicio (QoS), tales como, disponibilidad de los servicios [17], ancho de banda garantizado [18] y baja latencia como se dispone en las redes de conmutación de circuitos para las líneas arrendadas E1,T1,VC-1, STM-1,etc.

Las redes troncales IP a gran escala, actualmente instaladas en todo el mundo se basan en ATM; sin embargo, recientemente, se ha introducido MPLS-TP como tecnología de BackBone de alta velocidad, proporcionando capacidad de conmutación orientada a conexión basada en enrutamiento IP y en protocolo de señalización IP.

La principal diferencia con respecto a IP sobre ATM es la capacidad de gestión integrada de la capa 3 y de la capa 2 . La figura 14, ilustra la evolución de las tecnologías de transporte.

En [15] se propone una arquitectura de red para un servicio de línea arrendada, donde se plantea configurar el tráfico antes de ingresar al nodo MPLS-TP es decir, separar los datos control de los datos de transmisión a fin de obtener una disponibilidad de 99,999\%, una garantía de ancho de banda de $100 \%$ y una latencia máxima de $10 \mathrm{~ms}$ a través de 20 nodos de interconexión MPLS-TP.

La arquitectura de red propuesta en [15] se ilustra en la figura 15.

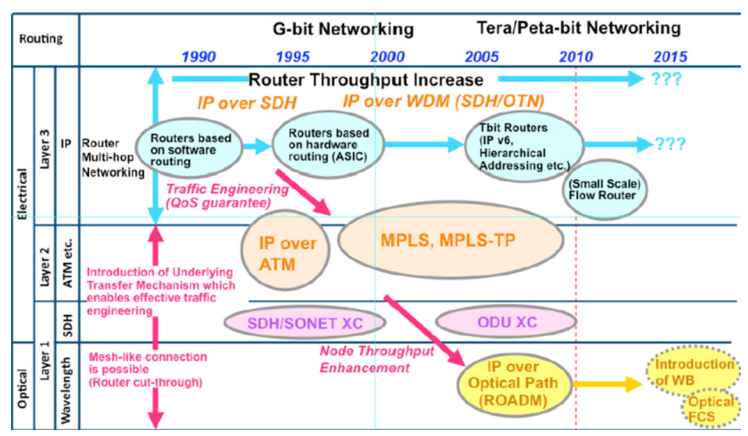

Figura 14. Evolución de las redes de transporte [14]

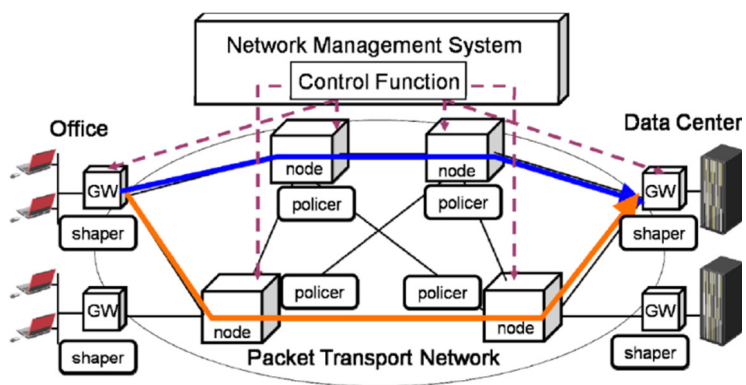

Figura 15. Arquitectura de red [15].

La investigación en [15] revela que es posible suprimir el tiempo de retardo a 0,5 ms si se realiza la configuración de los flujos de datos de usuario antes de ingresar al nodo de la red MPLS-TP; demostrando la posibilidad de garantizar un ancho de banda de $100 \%$, una la latencia máxima de $10 \mathrm{~ms}$ a través de 20 nodos MPLS-TP.

En [14] se propone una arquitectura de red jerárquica de tipo (HOXC), la cual busca reducir la escala de conmutación óptica OXC. La implementación de los componentes para una red en anillo y conexión del sistema HOXC se ilustra en la figura 16. 


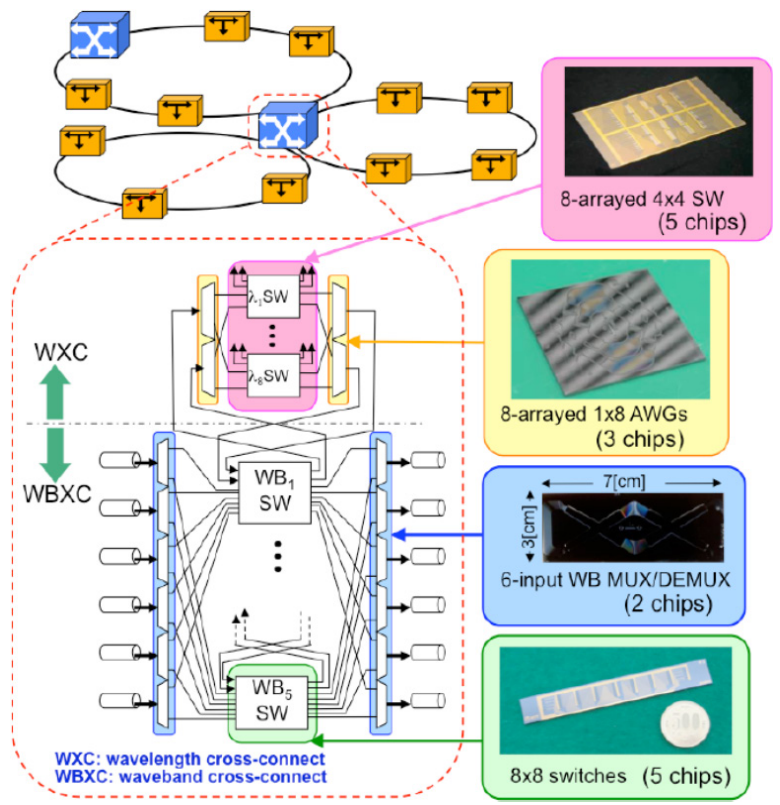

Figura 16. Aplicación de la conexión del sistema HOXC

[14]

Como resultado de la investigación en [14] la arquitectura jerárquica OXC (Optical CrossConnect) (HOXC) logra el 60\% de reducción en la escala de conmutación en comparación con la arquitectura de capa simple con la misma capacidad de enrutamiento; demostrando así ser satisfactoria para las implementaciones de MPLS-TP.

Investigaciones adicionales sobre el consumo energético se realizan en torno a los nuevos diseños de arquitecturas de redes de transporte ópticas; como se identifica en [14] y [16], el consumo de energía de las TIC's (Tecnologías de Información y Comunicación) será un factor apremiante en los diseños de las nuevas arquitecturas de red, máxime si se tiene en cuenta la importancia del consumo de energía de los routers con conexiones ópticas. En [14] se ha demostrado la mejora significativa de eficiencia energética y rendimiento mediante el uso de enrutamiento IP de capa 3 y conmutación de capa 2 como se ilustra en la figura 17.

En términos de eficiencia de energía y rendimiento, la conmutación de capa inferior es más eficiente, sin embargo, la capacidad de ancho de banda fijo no siempre es eficiente en comparación con la capacidad de ruta flexible de ancho de banda proporcionado por un LSP (Label Switching Path). Por lo tanto, las rutas TDM como VC (Virtual Containers) en SDH y ODU en OTN (Optical Transport Networks) están estructuradas jerárquicamente, los caminos de orden inferior ofrecen acceso a los servicios, mientras que los trayectos de orden superior proporcionan acceso a la transmisión [19], [20].

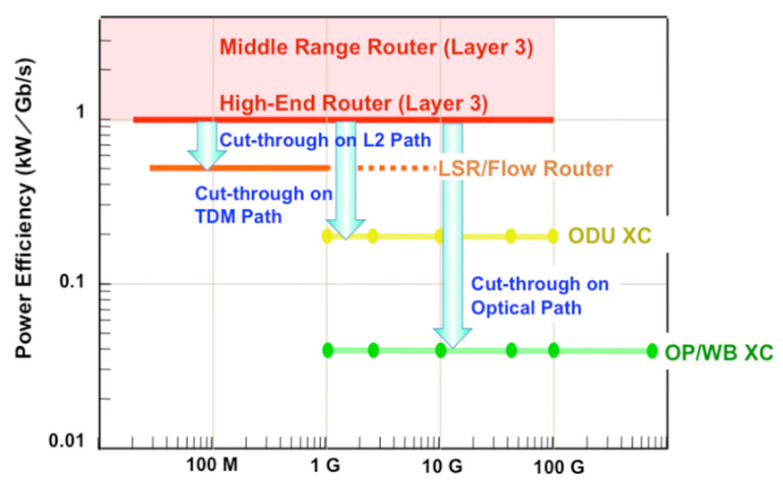

Figura 17. Evolución de los mecanismos de transporte IP [14]

\subsection{Protección OAM para MPLS-TP}

La tecnología MPLS-TP está ganando importancia, ya que se convierte en una solución dominante para una red de transporte convergente. Los mecanismos de protección se definen en los estándares a fin de proporcionar a la red la capacidad de restaurar la entrega del tráfico después de un fallo o degradación de los recursos. Las investigaciones que se realizan en [21], [22], [23], [24], [25], [26] y [27] están enfocadas a la implementación de nuevas metodologías que permitan reducir al máximo la pérdida de paquetes en comparación con los mecanismos de protección actualmente implementados para garantizar la fiabilidad de los servicios MPLS-TP.

La masificación de MPLS-TP como tecnología de transporte de paquetes ha llevado a la necesidad de generar un robusto conjunto de herramientas de protección. Existen en particular otras motivaciones para generar los mecanis- 
mos de protección para MPLS-TP, tales como:

1. La operación protección de la conmutación, es básicamente una capacidad del plano de datos dentro del contexto de MPLS-TP y necesita ser tan similares como sea posible a los sistemas de protección ya utilizados en las redes de transporte existentes como $\mathrm{SDH}$.

2. Existe una fuerte demanda de resiliencia (por ejemplo, 50ms de tiempo límite de restauración) en escenarios de protección.

3. Los mecanismos de protección deben proporcionar un buen comportamiento ante el incremento en el número de enlaces o vías de transmisión.

Los mecanismos de protección para MPLS-TP operan bajo el principio de dominios jerárquicos protegidos; En otras palabras, múltiples niveles de dominios protegidos se pueden manejar con un solo mecanismo de protección.

El dominio protegido es el conjunto de elementos de red al que se le puede proporcionar protección; este conjunto de elementos puede representar un lapso, una sección, o un trayecto de extremo a extremo; la figura 18, ilustra un modelo típico de referencia para un dominio jerárquico protegido.

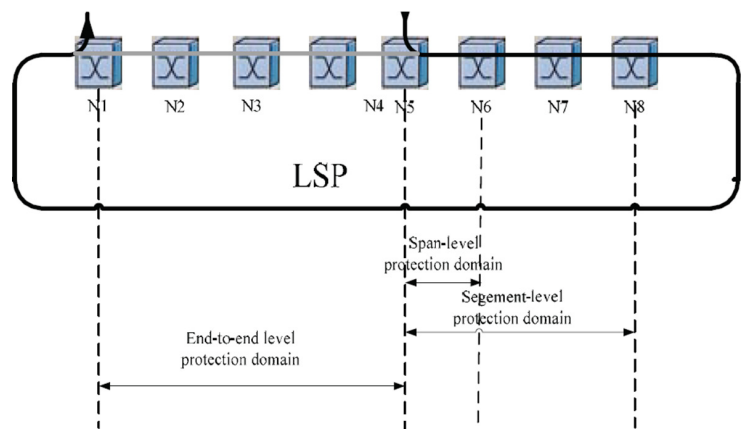

Figura 18. Modelo de referencia protección en anillo MPLS-TP [21]

En los RFC's de la IETF para protección de MPLS-TP [28], [29], se especifican dos arquitecturas de protección:

1. "wrapping" definido como un mecanismo de protección local el cual se implementa en un tiempo relativamente corto con menor perdida de paquetes [22].

2. "steering" definida como un mecanismo de protección en el cual cada nodo de origen y cada nodo receptor realizan el cambio de las conexiones MPLS-TP afectadas en el anillo de trabajo, a la dirección de protección [22]; en este esquema todos los nodos en el anillo deben analizar cual LSP han sido afectados por el fallo.

Mediante la combinación de "wrapping" y "steering", se obtiene un enfoque mejorado con un mejor rendimiento para evitar la pérdida de paquetes, mejorar el retardo y la eficiencia de ancho de banda disponible, se ejecutan principalmente dos pasos:

1. Cuando se detecta un fallo, "wrapping" se ejecuta inmediatamente para liberar el segmento de falla del anillo, cuyo mecanismo es el descrito para "wrapping". Este paso está dirigido a lograr la rápida conmutación de la protección y la consecuente menor pérdida de paquetes.

2. Seguidamente se ejecuta "steering" para proporcionar una ruta de protección optimizada en comparación con "wrapping". Este paso evita demoras innecesarias en la restauración del servicio y mejora la eficiencia de la red.

Un ejemplo de la combinación de la estrategia de combinación "wrapping" luego "steering" se ilustra en la figura 19.

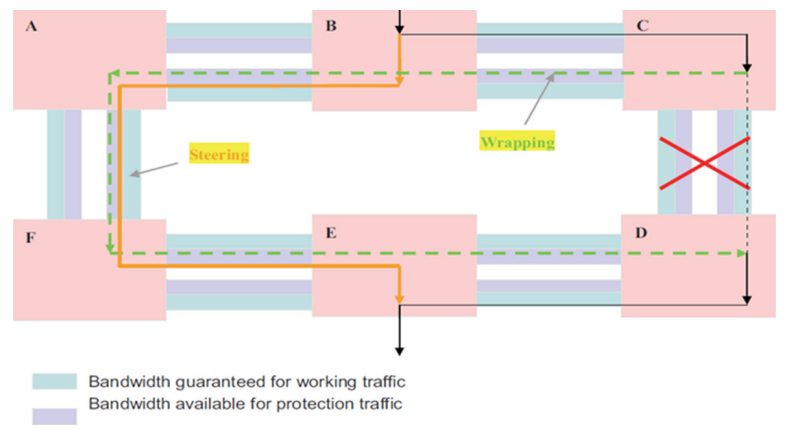

Figura 19. Esquema de protección de conmutación en MPLS-TP [21]

El LSP1 se establece entre el nodo B y E a través 
del nodo C y D. Cuando se detecta la falla o avería entre el nodo $\mathrm{C}$ y $\mathrm{D}$, inicialmente el tráfico normal es direccionado en primer lugar a la vía de protección entre $\mathrm{C}$ y $\mathrm{D}$ mediante la técnica de "wrapping" (línea punteada verde), para reducir la pérdida de paquetes como sea posible.

El nodo fuente B y el nodo receptor E del LSP1 a continuación realizan la conmutación mediante la técnica de "steering"; finalmente el tráfico es transmitido desde B a E a través de A y F (línea sólida naranja).

El mecanismo descrito anteriormente se conoce como "wrapping then steering", en el estándar RPR (Resilient Packet Ring) [30], el cual agrega lo mejor de ambas técnicas. En esta estrategia, se requiere de $\mathrm{O}\left(\mathrm{N}^{2}\right)$ rutas alternativas para cada anillo con nodos.

En [21] se propone un nuevo esquema de protección hibrido mejorado, donde la idea es mantener el orden estricto de los paquetes ante un eventual fallo en el anillo de fibra óptica, mientras se ejecuta la estrategia de mantenimiento "wrapping then steering" a fin de mantener la trayectoria del bucle de retorno con un marcador.

Se utiliza el formato APS MPLS-TP prolongado para indicar el marcador; así, el valor del campo "reservado" se cambia de "0x00" en "0x01" mientras que los otros campos de MPLS-TP APS PDU se mantienen sin cambios.

El formato de la APS (Automatic Protection Switching) extendida se ilustra en la figura 20.

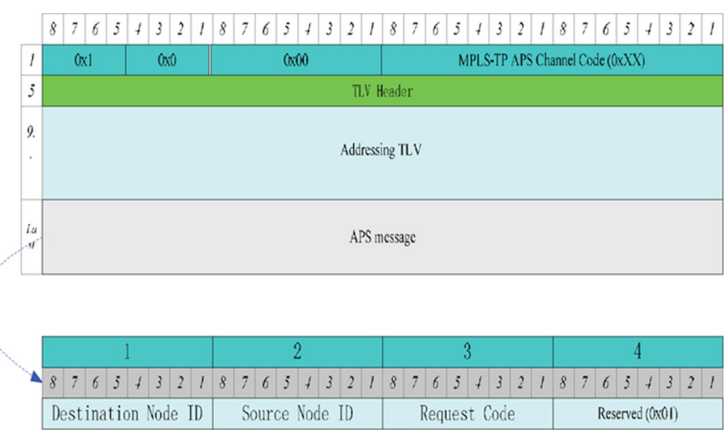

Figura 20. APS PDU extendida con información de marcador [21]
El mecanismo de [21] se compone de 4 pasos "wrapping" y "steering".

1. Cuando se detecta un fallo, "wrapping" se ejecuta inmediatamente para asilar la falla.

2. El nodo de origen genera una marca y la inserta después de que el paquete ha sido enviado a lo largo de la ruta de "wrapping" cuando el nodo fuente realiza la conmutación de finalización.

3. El nodo caído recibe el tráfico en la dirección de trabajo y descarta los paquetes en la dirección de protección, siempre y cuando el nodo receptor reciba el marcador en la dirección de trabajo. Una vez que el marcador llega al nodo receptor, el nodo receptor descarta todo el tráfico posterior en la dirección de trabajo y recibe el tráfico en dirección a la protección.

4. Finalmente se implementa "steering", así, el tráfico se transmite a lo largo de la ruta de protección de "steering".

Los resultados de la simulación de [21] se ilustra en la figura 21 donde se observa la pérdida de paquetes de un flujo de datos desde un nodo "0" para una estación de destino variada en un anillo de 64 estaciones en un escenario de avería.

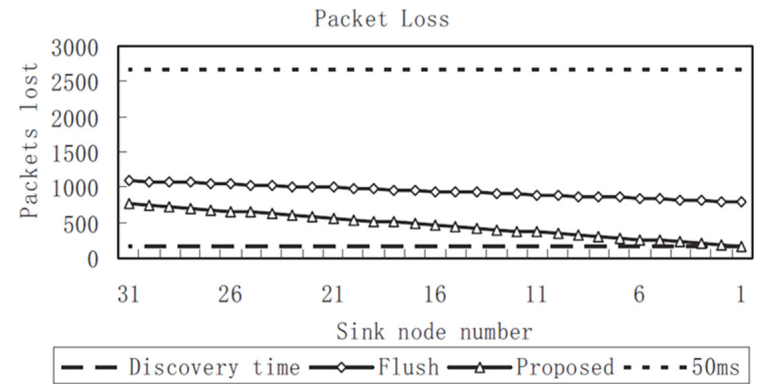

Figura 21. Pérdida de paquetes métodos convencional Vs hibrido [21]

La investigación en [21] revela que en el escenario de simulación la pérdida de paquetes se reduce entre un 30\% y hasta un 70\% comparado con el método convencional "wrapping" y "steering".

La reducción en la pérdida de paquetes está supeditada por los paquetes en tránsito a lo largo 
de la ruta de protección; por lo tanto, el rendimiento se mejora aún más cuando el nodo receptor está más cerca de la estación de origen.

La investigación realizada en [23] propone un nuevo esquema de protección mediante la utilización de un algoritmo de planificación de recursos que calcula la capacidad de reserva mínima requerida para conseguir la disponibilidad deseada de todas las rutas protegidas, así como la estrategia para la asignación de prioridades a las rutas de respaldo.

La investigación en [23] dispone el modelo planteado como una contribución al diseño de herramientas de ingeniería de tráfico para los servicios de LSP ofrecidos por las redes MPLSTP. Satisface algunos de los requisitos más importantes de la norma MPLS-TP emergentes, tales como el apoyo a la protección y los objetivos de disponibilidad definidos en los SLA's. Las pruebas realizadas con el modelo basado en el estado indican que, en algunos casos con prioridades distintas en los recursos de respaldo permite alcanzar el nivel deseado de protección con una economía significativa de ancho de banda.

En [22] se introduce la arquitectura de protección ECOFRAME para MPLS-TP inicialmente propuesta en [31], la cual se basa en la conmutación óptica de paquetes, tiempo fraccionado y un anillo WDM unidireccional con un solo canal de control fuera de banda. Los canales WDM son fraccionados en tiempo ya que es más eficiente administrar y gestionar paquetes de longitud fija que los paquetes de longitud variable [32] especialmente para fines de sincronización; por otra parte el tamaño de la ranura de tiempo está por el orden de los 10 micro segundos, que es significativamente más grande que el tamaño de un paquete de Ethernet, por lo tanto, la fragmentación no genera inconvenientes.

ECOFRAME permite la conmutación óptica transparente en el plano de datos. La información de control relativa a cada canal de datos y para el anillo global (por ejemplo, OAM) es también fraccionada en tiempo. La propuesta de [22] extiende la arquitectura ECOFRAME con anillos duales que permanecen coordinadas con el fin de permitir mecanismos que proporcionen resiliencia. La figura 22, muestra un anillo bidireccional basado en P-OADM WDM.

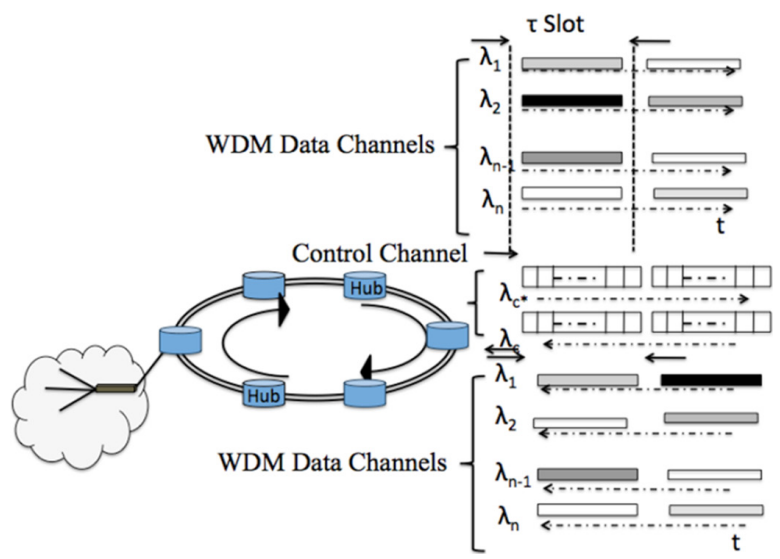

Figura 22. Arquitectura ECOFRAME de doble anillo [22]

Los nodos proporcionan granularidad de conmutación por sub-longitud de onda (es decir, a nivel de paquete) y transparencia óptica por medio de la separación de los canales de datos del canal de control. Las fibras de respaldo (de conmutación hacia atrás) proporcionan la capacidad de protección contra un fallo único en la red. Los dos canales de control fuera de banda se encuentran sincronizados con los canales de datos y llevan la información de OAM y de control relacionada con cada fibra de forma separada.

En [22] se describe el método de protección para anillos bidireccionales, el cual emula el clásico método de protección 1:1 usado en SDH [33].

En [24] se extiende este concepto a topologías multi-anillo, definiendo un camino etiquetado de protección para cada ruta de trabajo unidireccional. La figura 23, muestra un par remoto de nodos fuente / destino donde, en caso de fallo en cualquiera de las posiciones marcadas la dirección de protección es la ruta de acceso de la línea punteada.

El mensaje de fallo OAM generado en el anillo 1 llega a uno de los nodos de interconexión. Los 
nodos de interconexión pueden detectar si se lleva al menos una etiqueta de trayectoria del anillo adyacente, y al que se ve afectado por el fallo, si éste es el caso, el nodo interconectado propaga el mensaje de fallo OAM en ambas direcciones al anillo conectado.

A medida que el mensaje de fallo de OAM retransmitido identifica la ubicación de la interfaz que lo genera, todos los nodos que hacen parte de la trayectoria de origen que se ha visto afectada, evalúan la etiqueta a fin de reenviar el tráfico por la ruta de protección.

Una característica interesante que se observa en la figura 23 , es que la misma trayectoria de la etiqueta de protección puede utilizarse para todos los diferentes tipos de fallo único en cualquiera de los anillos, incluyendo la falla del nodo de interconexión. Esto implica que la tabla de etiquetas en cada nodo es fija, incluso durante un fallo transitorio.

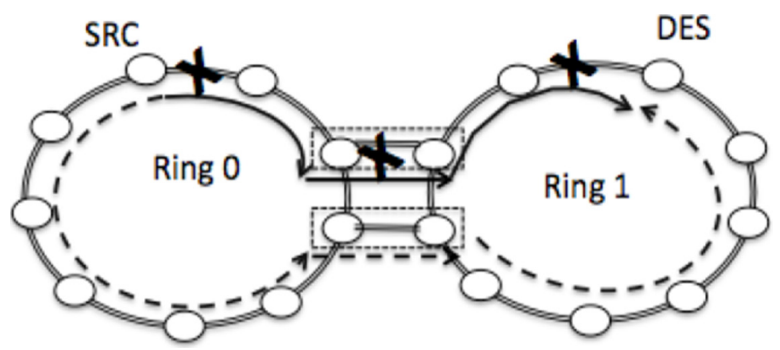

Figura 23. Protección regular en una red ECOFRAME multianillo [24]

En [22], se ha propuesto un método de protección segmentada para el tráfico de multidifusión en un solo anillo bidireccional, que se basa en la utilización de un MUD (Multicast Dropoff Point).

En un solo anillo, el MUD se encuentra frente a la fuente de multidifusión realizando dos funciones principales:

1. Desencapsular la PDU de multidifusión en modo nominal y reenviarla luego de que se haya marcado con el indicador $\mathrm{W} / \mathrm{B}$ en caso de falla.

2. Tal como se especifica en [24], se extiende este comportamiento para redes multi-anillo mediante la ampliación de los nodos de interconexión con los roles de desencapsulamiento y reenvió de PDU's de multidifusión.

En particular, esta tarea evita la definición de rutas de respaldo para el tráfico de multidifusión, y también el reenvió de información de falla de un anillo a otro, siempre y cuando únicamente la ruta etiquetada de multidifusión se vea afectada por el fallo.

Las investigaciones realizadas en [22] y [24] demuestran que la arquitectura ECOFRAME como estrategia de protección representa una opción atractiva para el apoyo de las funciones de protección OAM para MPLS-TP, con tiempos de recuperación de apenas unos pocos milisegundos.

\section{PERSPECTIVA DE MPLS-TP}

Por Conmutación por etiquetas multiprotocolo - perfil de transporte MPLS-TP, se entienden las extensiones del protocolo de conmutación por etiquetas multiprotocolo desarrollado por la Comisión de Estudio 15 del UIT-T en colaboración con el Grupo Especial sobre Ingeniería de Internet (IETF).

Muchos operadores de red esperan que MPLSTP funcione según los mismos principios que tecnologías de transporte de la UIT de larga data, como la jerarquía digital síncrona y la red óptica de transporte.

Los expertos del UIT-T y del IETF han mantenido prolongados debates en los últimos años. La Comisión de Estudio 15 ha remitido a la AMNT12 un importante proyecto de norma (UIT-T G.8113.1), un protocolo Ethernet para operaciones, administración y gestión de MPLS-TP para su aprobación, así como el proyecto de norma UIT-T G.8113.2 sobre "Mecanismos de operación, administración y mantenimiento de redes MPLS-TP utilizando las herramientas definidas para MPLS" [34]. 


\section{CONCLUSIONES}

MPLS-TP es una tecnología optimizada para transporte de paquetes, pero no se limita sólo a este fin; ofrece un camino para la evolución de las redes basadas en SONET/SDH a la conmutación de paquetes, manteniendo las tecnologías de circuitos de apoyo a través emulación mediante pseudo-cables.

Permite una convergencia entre los equipos y el backbone de transporte, proporcionando la flexibilidad y la escalabilidad requerida por Ethernet e IP, preservando al mismo tiempo la capacidad de gestión, capacidad de recuperación, el rendimiento determinista y funciones OAM de las redes de transporte tradicionales.

Las recientes investigaciones sobre MPLS-TP se están enfocando en gran medida a desarrollos de nuevos modelos de arquitecturas ópticas que permitan el despliegue de velocidades superiores a los $100 \mathrm{Gbps}$ a un costo financiero y energético rentable; el énfasis que se realiza en la protección OAM para MPLS-TP ha generado varios estudios donde se busca la mayor opor-

Referencias Bibliográficas

[1] International Telecommunication Union - Study Group ITU-T Newslog. [En linea], consultado en Marzo 15 del 2013, disponible en: http://www.itu.int/ITU-T/ newslog/MPLSTP+The+Facts.aspx.

[2] O. Corrigent. [En linea], consultado en Enero 20 del 2013, disponible en:http:// www.orckit.com/\&mod=download\&tab le $=$ stories $1 \&$ field=st_pdf1\&me_id=107.

[3] R. Ankur, P. Sasindran; MPLS Evolution: MPLS Transport Profile. [En linea], consultado en Octubre 3 del 2012, disponible en:http://www.techmahindra. com / Documents / WhitePaper/ MPLSTPWhitepaper.pdf

[4] W. Stallings; Comunicaciones y Redes de Computadores, Sexta edición, Editorial MCGraw Hill, 2000.

[5] S. Chen; sitio web de MPLS Japan. [En tunidad de resiliencia a fin de lograr la menor perdida de paquetes, particularmente con los servicios que emulan líneas dedicadas arrendadas de alta capacidad.

MPLS-TP se perfila como la red de transporte de paquetes de nueva generación; la agregación de las mejores características de las redes de transporte ópticas para conmutación de paquetes (IP) y conmutación de circuitos (TDM) permite la convergencia de múltiples plataformas y servicios.

Las principales ventajas radican en las funciones y operaciones de OAM a través de las diferentes capas de red además de su compatibilidad e interfuncionamiento con redes IP/MPLS. MPLS-TP es altamente escalable debido a su capacidad de Multiplexación que se puede utilizar para crear una red con múltiples capas jerárquicas. MPLS-TP es compatible con una gran variedad de servicios que están encapsulados en pseudo-cables y se puede portar a través de las redes de transporte existentes y en evolución de infraestructura.
[6] K. Christos; Multi Protocol Label Switching Transport Profile (MPLS-TP) in OpMiGua Hybrid Network. MSc Tesis, Norwegian University of Science and Technology (NTNU), 2010.

[7] D. Beller, S. Rolf. [En linea], consultado en Octubre 10 del 2012, disponible en https://www.dfn.de/fileadmin/3Beratung/ DFN-Forum2/118.pdf

[8] International Telecommunication Union; Key Results of the Work on MPLSTP. [En linea], consultado en Marzo 10 del 2013, disponible en: http:// www.itu.int/dms_pub/itu-t/oth/09/06/ 
T09060000010002MSWE.doc

[9] O. Pinzon, E. Sierra. [En linea], consultado en Octubre 17 del 2012, disponible en: http://es.scribd.com/doc/62234395/ Estado-Del-Arte-Mpls-TP

[10] Juniper Networks. [En linea], consultado en Octubre 17 del 2012, disponible en: http://www.juniper.net/us/en/local/pdf/ whitepapers/2000406-en.pdf

[11] International Telecommunications Union, Recommendation ITU-T G.8110.1/Y.1370.1 Architecture of the Multi-Protocol Label. [En linea], consultado en Octubre 17 del 2012, disponible en: http://www.itu.int/rec/TREC-G.8110.1.

[12] Internet Engineering Task Force, Requirements of an MPLS Transport Profile - RFC5654. [En linea], consultado en Octubre 17 del 2012, disponible en: http://datatracker.ietf.org/doc/rfc5654/

[13] F. Hans; ASON/GMPLS control plane solutions for transport networks, IEEE Xplore Digital Library, pp 1-2, 2008.

[14] S. Ken-ichi; Next Generation Transport Network Architecture, IEEE Xplore Digital Library, pp 1-3, 2010.

[15] O. Takumi, T. Masayuki, S. Kenichi, E. Hideki: Implementation of packet transport system using MPLS-TP Technologies, IEEE Xplore Digital Library, pp 1-6, 2010.

[16] S. Hauger; Packet processing at 100 gbps and beyondchallenges and perspectives, IEEE Xplore Digital Library, pp 1-10, 2009.

[17] D. Xu, Y. Li, M. Chiang, A. Calderbank; Elastic service availability: utility framework and optimal provisioning, IEEE Journal, vol. 26, no. 6, pp 78-75, 2008.

[18] A. Berger, W. Whitt; Extending the effective-bandwidth concept to networks with priority classes, IEEE Communications Magazine, vol. 36, no. 8, pp 78-84, 1998.

[19] K. Sato; Advances in transport network technologies: photonic networks, ATM, and SDH, 1996.

[20] K. Sato. H. Hasegawa; Prospects and challenges of multi-layer optical networks, IEICE Trans. Commun., vol. E90-B, no. 8, pp 1890-1902, 2007.

[21] W. Xie, S. Huang, W. Gu; An improved ring protection method in MPLS-TP networks, IEEE International Conference, pp 1-5, 2010.

[22] L. Sadeghioon, A. Gravey, P. Gravey; Rapid Protection Schemes in an AllOptical Packet Metro Ring, IEEE Xplore Digital Library, pp 1-6, 2012.

[23] E. Jamhour, M. Penna; a state-based availability model to shared mesh, Network Operations and Management Symposium (NOMS), pp 926-932, 2012.

[24] L. Sadeghioon, P. Gravey, A. Gravey, Resilience in transparent OPS multi-rings, Optical Network Design and Modeling (ONDM), pp 1-3, 2011.

[25] J. Zhang, S. Ruepp, M. Berger, H. Wessing; Protection for MPLS-TP multicast services, IEEE Conference Publications, pp 297-304, 2009.

[26] J. Zhang, M. Berger, S. Ruepp; Flowbased end-to-end oam functions for the multicast service on the MPLS-TP ring network, Communications (ICC), pp 1-5, 2010.

[27] J. Zhang; Two novel tunnel-based ring protection switching for MPLS-TP multicast services, IEEE Telecommunications (ICT), pp 458-462, 2011.

[28] Y. Weingarten; MPLS-TP ring protection, IETF MPLS Working Group. [En linea], consultado en Noviembre 4 del 2012, disponible en: http://tools.ietf.org/ $\mathrm{html} / \mathrm{draft}$-weingarten-mpls-tp-ringprotection-06

[29] I. Umansky; MPLS-TP ring protection switching, IETF MPLS Working Group. [En línea], consultado en Noviembre 4 del 2012, disponible en: http://tools. ietf.org/id/draft-umansky-mpls-tp-ringprotection-switching-03.txt

[30] IEEE, Resilient packet ring, IEEE standard 802.17. [En línea], consultado en Noviembre 4 del 2012, disponible en: http://www.nanog.org/meetings/nanog27/ presentations/brown.pdf 
[31] D. Chiaroni; Demonstration of the interconnection of two optical packet rings with a hybrid optoelectronic packet router european, Conf. on Opt. Commun., vol. PD3, no. 5, pp 340-345, 2010.

[32] J. Li, C. Qiao, J. Xu, D. Xu; Maximizing throughput for optical burst switching networks, IEEE/ACM Transactions On Networking, vol. 15, no. 5, pp 78-80, 2011.

[33] International Telecommunications Union, Recommendation G.841, Types and characteristics of SDH network, Recomendación 1995. [En línea], consultado en Octubre 20 del 2012, disponible en: http:/www.ie.itcr.ac.cr/ jstradi/Comunicaciones/Cables_Sub/ g841_e_2.pdf

[34] International Telecommunication Union. [En linea], consultado en Octubre 27 del 2012, disponible en: https://itunews. itu.int/es/3378-Logros-de-los-ultimoscuatro-anos.note.aspx 